\title{
Intraoperative indocyanine green fluorescence lymphography to detect chylous leakage sites after congenital heart surgery
}

\author{
Te-I Chang, MD, Yih-Sharng Chen, MD, PhD, and Shu-Chien Huang, MD, PhD, Taipei, Taiwan
}

Chylothorax is a well-known complication that can occur after complex congenital heart surgery. The majority of patients can be treated by conservative methods, such as diet modification, total parenteral nutrition, and octreotide therapy. ${ }^{1}$ A small number of patients who do not respond to medical treatment will require surgical thoracic duct ligation. However, there are not many reports discussing solutions for refractory chylothorax after failure of surgical treatment. We describe a novel technique by incorporating intraoperative indocyanine green (ICG) fluorescence lymphography to detect chylous leakage. By subcutaneous injection of ICG at the groin region, we successfully detected and sutured a chylous leakage site through

From the Department of Surgery, National Taiwan University Hospital, National Taiwan University College of Medicine, Taipei, Taiwan.

This study was partly supported by NSC grants 102-2325-B-002 -036 and 102-2314B-002 -032 from the National Science Council, Taiwan.

Disclosures: Shu-Chien Huang reports consulting fees from Baxter. All other authors have nothing to disclose with regard to commercial support.

Received for publication Dec 23, 2013; revisions received Feb 27, 2014; accepted for publication March 14, 2014; available ahead of print April 13, 2014

Address for reprints: Shu-Chien Huang, MD, PhD, Department of Surgery, National

Taiwan University Hospital, 7 Chung-Shan South Rd, Taipei 100, Taiwan (E-mail:

cvshuang@gmail.com).

J Thorac Cardiovasc Surg 2014;148:739-40

$0022-5223 / \$ 36.00$

Copyright (C) 2014 by The American Association for Thoracic Surgery

http://dx.doi.org/10.1016/j.jtcvs.2014.03.021 re-sternotomy in a 3-month-old infant with congenital heart disease who had postoperative refractory chylothorax despite numerous medical and surgical managements. The chylous drainage ceased promptly after the operation, and the method was shown to be precise and specific.

\section{CLINICAL SUMMARY}

A full-term male infant was diagnosed with an endocardial cushion defect, severe coarctation of the aorta, a large patent ductal arteriosus, and an aberrant right subclavian artery. A series of operations, including aortoplasty, patent ductal arteriosus division, and main pulmonary artery banding, were undertaken after birth. Total corrective surgery of the endocardial cushion defect was then performed once the boy reached 3 months of age. The boy presented with increased chylothorax after the surgery and did not improve despite fasting and total parenteral nutrition support. Two thoracotomy approaches of thoracic duct ligation from the right and left sides were tried but failed to reduce the chylous drainage (Figure 1).

The chylothorax output remained for weeks without improvement. To locate the chylous leakage point, we decided to reexplore the mediastinum on postoperative day 45. Informed consent was obtained from the patient's family, and we adopted the novel technique of intraoperative ICG fluorescence lymphography.

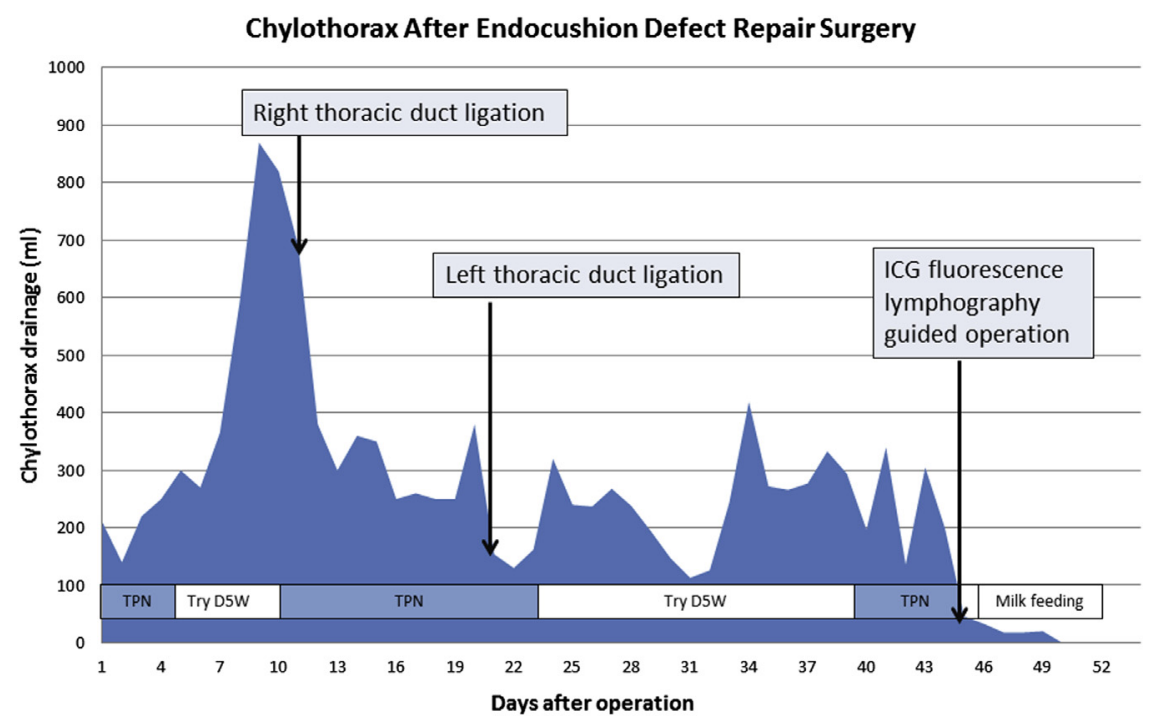

FIGURE 1. Daily chylothorax drainage amount after endocardial cushion defect surgery. The horizontal bar indicates the time periods we administered total parenteral nutrition, $5 \%$ dextrose water, or milk feeding. $D 5 \mathrm{~W}, 5 \%$ dextrose water; $I C G$, indocyanine green; $T P N$, total parenteral nutrition. 


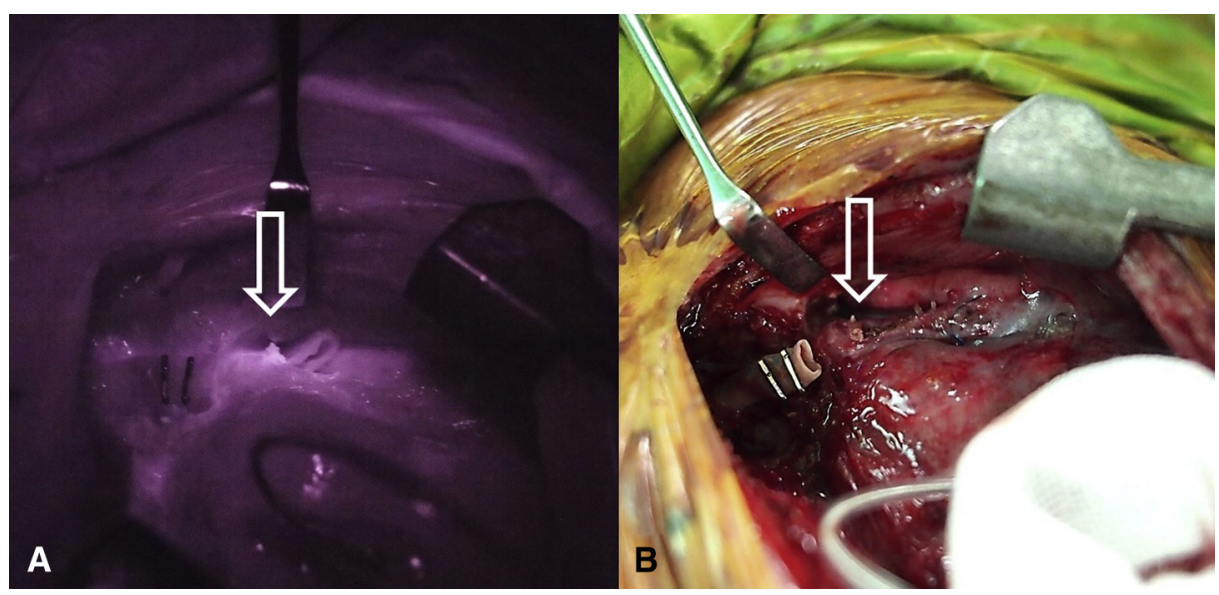

FIGURE 2. Intraoperative images showing (A) fluorescence lymphography view with a white arrow identifying a hotspot of ICG leakage after sternotomy. $\mathrm{B}$, Color image of the same field with a white arrow indicating the leakage fistula. The patient's head is to the left.

After resternotomy, we found no visible chylous leakage despite meticulous examination. Intraoperative fluorescence lymphography was then performed by injecting $2 \mathrm{~mL}$ of ICG (Diagnogreen 0.5\%; Daiichi Pharmaceutical, Tokyo, Japan) subcutaneously at the bilateral inguinal region for absorbance into the lymphatic system. An infrared camera and a near-infrared excitation light (light-emitting diode $740 \mathrm{~nm}$ ) were used to capture the ICG fluorescence signal image. The inguinal region glowed immediately after the injection. After approximately 20 minutes, we noticed a fluorescent hotspot that coincided with a fistula-like structure at the lateral side of the aorta (Figure 2, $A$ and $B$ ). The fistula was recognized to be a major chylous leakage point, and after ligation with a pledgeted 4-0 Prolene suture, there was no further accumulation of lymphatic ICG. The absence of further leakage was confirmed by applying dry gauze to the field and checking for any residual ICG stain on the gauze using the near-infrared camera. The chylothorax drainage ceased after the operation. There was no chylothorax recurrence after the patient began formula feeding, and his chest tubes were removed 1 week later.

\section{DISCUSSION}

ICG is a hydrophilic tricarbocyanine dye that rapidly binds to plasma proteins in the body and is mostly incorporated into the liver and excreted in the bile. When ICG in the blood is exposed to a near-infrared ray of 760 to $780 \mathrm{~nm}$ wavelength, it generates fluorescence of 800 to $850 \mathrm{~nm}$ wavelength. ${ }^{2}$ Drug safety is well established with few side effects. The dye contains sodium iodide and should be used with caution in patients who are allergic to iodides.

ICG was first used as a fluorescence dye in angiography, but it has also proven to be advantageous for use in lymphography because of its highly sensitive fluorescence properties. Hojo and colleagues ${ }^{3}$ demonstrated a single dose of $2 \mathrm{~mL}$ ICG subcutaneous injection for identification of sentinel lymph node in breast cancer surgery.

To locate an injured chylous duct, lymphoscintigraphy with technetium- $99 \mathrm{~m}$ can be considered, ${ }^{4}$ but this method does not allow real-time imaging or pinpointing of the exact leakage site. A preoperative oral ingested heavy cream method also may be used to identify thoracic lymph ducts; however, this method provided poor contrast results in comparison with ICG fluorescence lymphography in this animal model.

\section{CONCLUSIONS}

Octreotide therapy has been used for chylothorax, but it is not covered by Taiwan's National Health Insurance. The new experimental ICG application in this case proved to be a precise tool for identification of a chylous leakage site with great sensitivity and specificity. It has good potential as an alternative solution in the treatment of patients with postoperative refractory chylothorax.

\section{References}

1. Tatar T, Kilic D, Ozkan M, Hatipoglu A, Aslamaci S. Management of chylothorax with octreotide after congenital heart surgery. Thorac Cardiovasc Surg. 2011;59: 298-301

2. Benson RC, Kues HA. Fluorescence properties of indocyanine green as related to angiography. Phys Med Biol. 1978;23:159-63.

3. Hojo T, Nagao T, Kikuyama M, Akashi S, Kinoshita T. Evaluation of sentinel node biopsy by combined fluorescent and dye method and lymph flow for breast cancer. Breast. 2010;19:210-3.

4. Baulieu F, Baulieu JL, Mesny J, Ducouret N, Benhamou AC, Barsotti J, et al. Visualization of the thoracic duct by lymphoscintigraphy. Eur J Nucl Med. 1987;13: 264-5

5. Ashitate Y, Tanaka E, Stockdale A, Choi HS, Frangioni JV. Near-infrared fluorescence imaging of thoracic duct anatomy and function in open surgery and video-assisted thoracic surgery. J Thorac Cardiovasc Surg. 2011;142:31-8, e1-2. 